\title{
ANÁLISE COMPARATIVA DE TRÊS TIPOS DE VÁLVULAS ANTI-REFLUXO ASSOCIADA À CIRURGIA DE HELLER: ESTUDO EXPERIMENTAL EM CÃES
}

\section{COMPARATIVE ANALYSIS BETWEEN THREE TYPES OF ANTI-REFLUX VALVES ASSOCIATED WITH HELLER'S SURGERY: EXPERIMENTAL STUDY IN DOGS}

\author{
Eduardo Crema, TCBC-SP ${ }^{1}$ \\ Daniela A. Ferreira ${ }^{2}$ \\ Alex A.Silva ${ }^{3}$ \\ Ruy Ferreira-Santos, ECBC-SP ${ }^{4}$
}

\begin{abstract}
RESUMO: Objetivo: Comparar experimentalmente, em cães, três técnicas de esofagogastrofundoplicatura anterior, posterior e lateral - após cardiomiotomia ampla. Método: Os animais foram separados aleatoriamente em quatro grupos: Grupo A: Válvula anti-refluxo anterior $(n=5)$; Grupo P: válvula anti-refluxo posterior $(n=5)$; Grupo L: Válvula anti-refluxo lateral $(\mathrm{n}=5)$; Grupo C: Grupo controle, no qual não foi realizado nenhum tipo de fundoplicatura após a cardiomiotomia $(\mathrm{n}=10)$. A avaliação pré-operatória foi realizada através de endoscopia, biópsia da mucosa gastroesofágica, pHmetria e manometria esofágica. Após cinco semanas, realizou-se reavaliação, baseada em dados endoscópicos, pHmétricos, manométricos e histopatológicos. Os animais foram mortos e os segmentos esofagogástricos retirados para exame histopatológico Resultados: Ao exame histopatológico detectou-se esofagite em todos os casos do grupo controle e em nove casos dos grupos com válvulas, e nestes, quando presente, a esofagite foi de menor extensão e de grau menos avançado do que no grupo controle. Através da manometria pré e pós-operatória, observou-se que: 1) o grupo controle apresentou uma redução na pressão na ZAP de 39,2mmHg para 23,4mmHg; 2) os grupos A, P, e L apresentaram uma variação da ZAP de 39,7, 39,9 e 39,7 mmHg para 40,9, 44,8 e 41,3mmHg, respectivamente. Conclusões: 1) A cardiomiotomia ampla provoca refluxo gastroesofágico e conseqüente esofagite; 2) As três válvulas anti-refluxo estudadas restauram o segmento de alta pressão e diminuem a incidência de refluxo e de esofagite.
\end{abstract}

Descritores: Esofagogastrofundoplicatura; Cardiomiotomia; Válvula anti-refluxo.

\section{INTRODUÇÃO}

Heller ${ }^{1}$ para tratar um paciente denominado por ele portador de cardioespasmo crônico, praticou pela primeira vez a cardiomiotomia extramucosa realizando duas incisões longitudinais, uma anterior e outra posterior, sobre a junção esofagogástrica, utilizando acesso abdominal através de incisão subcostal.

Groeneveldt $^{2}$, em 1918, modificou a técnica original, efetuando a cardiomiotomia somente sobre a face anterior, com resultados pós-operatórios semelhantes aos obtidos por Heller. Esta operação sofreu inúmeras modificações, mas conserva o nome do autor, sendo denominada cardiomiotomia de Heller.

No Brasil, Seng ${ }^{3}$ foi o primeiro a realizar esta intervenção, datando de 1922 a publicação de sua experiência com o uso da técnica de Heller modificada, para o tratamento do megaesôfago chagásico.

Mattos ${ }^{4}$, em 1938, propôs a cardiomiectomia longitudinal com retirada de uma fita muscular da transi-

1. Professor Titular e Responsável pela Disciplina de Cirurgia do Aparelho Digestivo (DCAD) da Faculdade de Medicina do Triângulo Mineiro (FMTM)

2. Residente de Cirurgia Geral - FMTM

3. Professor Adjunto da DCAD-FMTM

4. Professor Catedrático Emérito (Departamento de Cirurgia) da Faculdade de Medicina de Ribeirão Preto-USP.

Recebido em 10/02/1999

Aceito para publicação em 11/06/2002

Trabalho realizado na Faculdade de Medicina do Triângulo Mineiro (FMTM). 
ção esofagogástrica, com o intuito de afastar as bordas da miotomia.

Notou-se, no entanto, o aparecimento pós-operatório de refluxo gastroesofágico (RGE), com incidência variável entre $18 \%$ a $45 \%$ e conseqüente esofagite de refluxo verificada em $10 \%$ a $20 \%$ dos casos devido à secção da zona de alta pressão (ZAP), localizada na transição esofagogástrica ${ }^{5-10}$.

Com o intuito de evitar o refluxo gastroesofágico procurou-se associar procedimentos anti-refluxo às técnicas de cardiomiotomia ${ }^{6,11,12,17}$. Entre as técnicas anti-refluxo, as gastrofundoplicaturas, totais ou parciais, são as mais utilizadas.

Nissen, em 1956, descreveu um procedimento técnico que consistiu em envolver o esôfago abdominal com o fundo gástrico, formando um manguito de $360^{\circ} \mathrm{em}$ torno do esôfago. Resultados satisfatórios no sentido de evitar o RGE, utilizando a técnica de Nissen, foram observados experimentalmente ${ }^{11}$ e clinicamente ${ }^{12,13}$. Contudo, a quase totalidade dos serviços propõe a utilização de válvulas parciais após as miotomias, visto que estes pacientes apresentam esôfagos com graus variáveis de dismotilida$\mathrm{de}^{14-17}$.

Jeckler e Lhotka ${ }^{18}$, em 1967, relataram resultados satisfatórios no acompanhamento clínico e radiológico pósoperatórios, em um período de seis a 40 meses, utilizando a proposta de Dor ${ }^{19}$.

Goffi ${ }^{15}$ propôs uma modificação da técnica de Lhotka-Dor ${ }^{18,19}$, envolvendo os $2 / 3$ anterolaterais do esôfago, produzindo assim uma válvula parcial anterior de $270^{\circ}$. Lind et al. ${ }^{20}$, em 1965, propuseram para o tratamento do RGE, uma modificação na técnica de Belsey, onde, com pontos transversais de fixação, o fundo gástrico envolve posteriormente o esôfago abdominal produzindo uma válvula parcial posterior de $270^{\circ}$.

Pinotti et al..$^{21}$, em 1974, preconizaram a associação do procedimento de Lind $^{20}$ nos pacientes submetidos a cardiomiotomia no tratamento do megaesôfago chagási- co. A sua modificação técnica consiste em que a sutura do fundo gástrico na borda lateral esquerda do esôfago seja feita na borda esquerda da miotomia. Acrescenta também uma terceira linha de sutura do fundo gástrico à borda lateral direita da miotomia, obtendo, desta forma, a cobertura de toda a superfície cruenta esofagogástrica. Este procedimento tem sido empregado de rotina em diversos serviços, com bons resultados pós-operatórios.

Frente às diversas modificações técnicas que sofreram as válvulas anti-refluxo e pela dificuldade de seu estudo comparativo em clínica, propusemo-nos estudar, em cães, três tipo de gastrofundoplicaturas parciais - anterior, lateral e posterior - depois de efetuada a cardiomiotomia ampla.

\section{MÉTODO}

O experimento foi realizado em 25 cães. Em todos foi feita miotomia longitudinal anterior de aproximadamente $8 \mathrm{~cm}$ na região da cárdia, sendo $5 \mathrm{~cm}$ no esôfago e $3 \mathrm{~cm}$ no estômago, seccionando toda a camada muscular até a obtenção de abaulamento franco da mucosa em toda a extensão. Após a miotomia, os animais foram sorteados e distribuídos em quatro grupos:

\section{Grupo A}

Válvula anterior: composto por cinco cães, nos quais se realizou esofagogastrofundoplicatura (EGFP) anterior com duas fileiras longitudinais de quatro pontos separados, com fio de algodão 000 , nas bordas lateroposteriores direita e esquerda do esôfago, cobrindo os seus 3/4 anteriores e laterais numa extensão de aproximadamente $6 \mathrm{~cm}$, formando um arco anterior de $270^{\circ}$ (Lhotka-Dor-Goffi) (Figura1).

\section{Grupo P}

Válvula posterior: composto por cinco cães, nos quais se realizou EGFP posterior com duas fileiras longitudi-

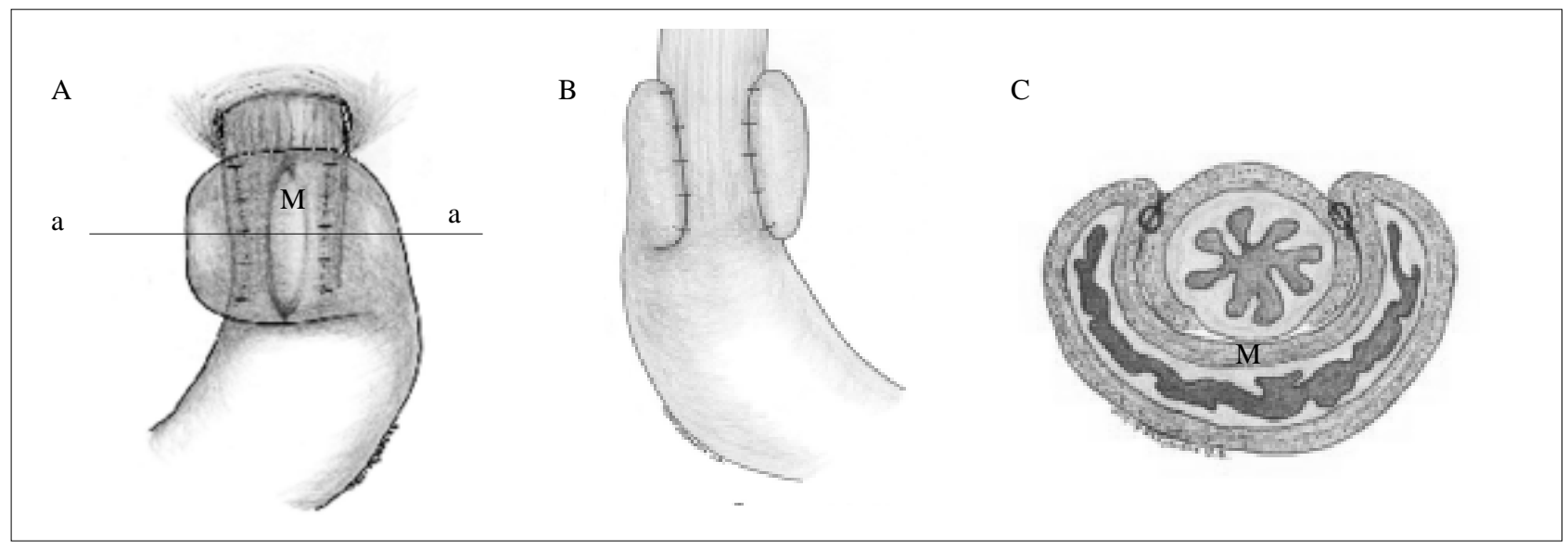

Figura 1 - Lhotka-Dor-Goffi. A) Vista anterior, mostra o envolvimento anterolateral do esôfago cobrindo totalmente a área de miotomia (M). B) Vista posterior, mostra os pontos dados póstero-lateralmente do esôfago, ficando somente o 1/4 posterior do esôfago descoberto. $C) O$ corte transversal $(a-a)$ mostra o fundo gástrico envolvendo o esôfago anterolateralmente formando um arco anterior de $270^{\circ}$ - nota-se área da miotomia $(M)$. 
nais de quatro pontos separados, com fio de algodão 000 , nas bordas látero-anteriores direita e esquerda do esôfago, envolvendo seus $3 / 4$ posteriores e laterais numa extensão de aproximadamente $6 \mathrm{~cm}$, formando um arco posterior de $270^{\circ}$ (Lind modificada) (Figura 2).

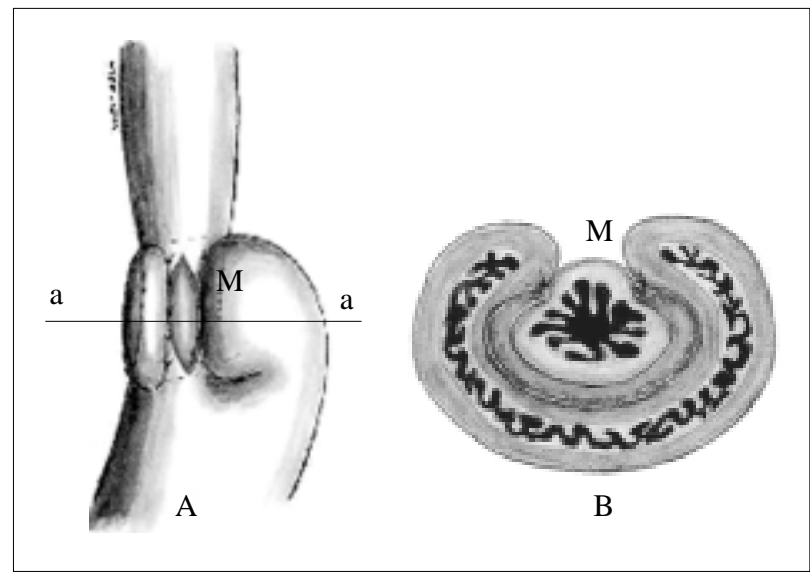

Figura 2 - Lind modificado. A) Vista anterior, mostra o envolvimento póstero-lateral do esôfago, nota-se área da miotomia $(M)$. B) $O$ corte transversal $(a-a)$ mostra o fundo gástrico envolvendo o esôfago póstero-lateralmente formando um arco posterior de $270^{\circ}$ - nota-se área da miotomia $(M)$.

\section{Grupo L}

Válvula lateral: composto por cinco cães, nos quais se realizou EGFP lateral com duas fileiras longitudinais de quatro pontos separados, com fio de algodão 000, envolvendo a metade lateral esquerda do esôfago, numa extensão de aproximadamente $6 \mathrm{~cm}$, formando um arco lateral de $180^{\circ}$ (Lortat-Jacob-Ferreira-Santos) (Figura 3).

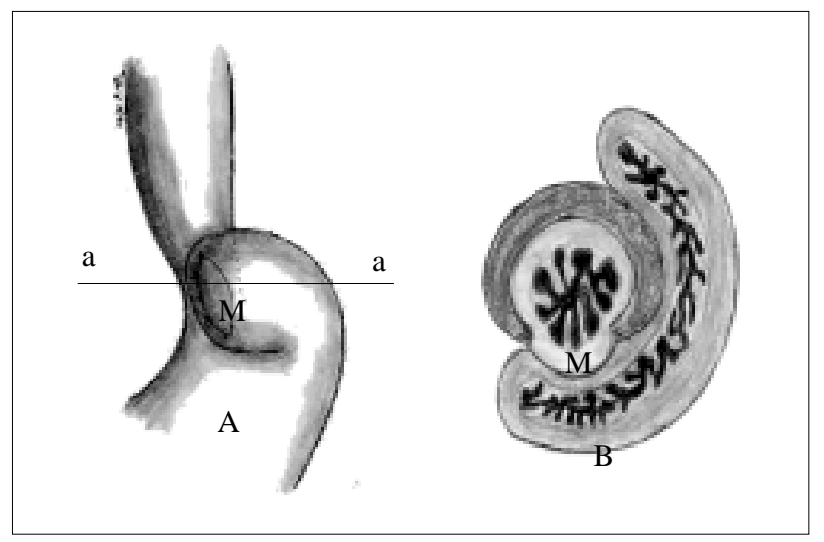

Figura 3 - Lortat-Jacob-Ferreira-Santos. A) Vista anterior, mostra o envolvimento lateral esquerdo do esôfago cobrindo a área de miotomia $(M)$. B) $O$ corte transversal $(a-a)$ mostra o fundo gástrico envolvendo o esôfago lateralmente à esquerda formando um arco lateral de $180^{\circ}$ - nota-se área da miotomia (M).

\section{Grupo C}

Sem válvula: composto por dez animais, nos quais, depois de completada a miotomia, não se confeccionou válvula anti-refluxo. Estes animais serviram como controle.

Para apreciação comparativa das três técnicas operatórias empregaram-se pré e pós-operatoriamente $\left(35^{\circ} \mathrm{PO}\right)$ os seguintes métodos:

1. Endoscopia: realizada com os animais sob anestesia geral endovenosa (Tiopental sódico na dose de $25 \mathrm{mg}$ / $\mathrm{kg}$ de peso), utilizando o fibroendóscopio GIFK.

2. Teste de refluxo ácido: realizado segundo a técnica preconizada por Pinotti.

3. Eletromanometria da transição esofagogástrica: realizada com a utilização de sonda de polivinil, registrador Physiograph MK IV, três pré-amplificadores e três tradutores de pressão ERP 1500, cuja relação de volume/pressão é de $0,009 \mathrm{~mm}^{3} / 100 \mathrm{mmHg}$, sistema hidráulico de perfusão (bomba de compressão e aspiração), 1 frasco de vidro de 1 litro de capacidade e tubos de polietileno PE 20.

4. Histopatologia dos fragmentos de biópsia endoscópica: os fragmentos da mucosa esofágica colhidos por endoscopia foram imediatamente fixados em formol neutro a $10 \%$ e após 48 horas de fixação foram processados para exame histológico. $\mathrm{O}$ critério utilizado para diagnosticar a esofagite e suas variações de intensidade foi resultado da análise e interpretação das alterações arquiteturais, estruturais e citológicas do epitélio esofágico.

5 Estudo macro e microscópico do segmento esofagogástrico estirpado no 35 dia de pós-operatório quando do sacrifício dos cães: As peças contendo esôfago terminal e estômago foram abertas pela grande curvatura e fixadas em formol a 10\%. Após 48 horas de fixação, as peças foram recortadas, obtendo-se fragmentos de aproximadamente $2 \times 1 \times 0,5 \mathrm{~cm}$, os quais, depois de devidamente identificados, foram desidratados em sentido crescente de álcoois, diafanizados, impregnados e emblocados em parafina. Após corados, os cortes foram analisados segundo os critérios histológicos de Ismail-Beige.

\section{RESULTADOS}

O teste de refluxo ácido foi positivo em sete animais do grupo controle, em dois animais do grupo com válvula anterior e em dois pertencentes ao grupo com válvula lateral. Em todos os cães com válvula posterior o teste foi negativo. Entretanto, quando comparados os grupos com válvulas entre si, as diferenças não foram estatisticamente significativas. Reunidos os grupos com válvula (A, P e L) e comparados ao grupo controle, foi significativa a diferença em relação ao teste positivo para o refluxo ácido.

O estudo manométrico não revelou diferença quanto à localização, pressão e extensão na zona de alta pressão (ZAP), quando os grupos foram comparados entre si no pré-operatório. A média das pressões na ZAP, no pré-operatório dos animais do grupo controle, foi de 
$39,2 \mathrm{mmHg}$, reduzindo-se significantemente no pós-operatório para $23,4 \mathrm{mmHg}$. Houve, pois, uma diferença de $15,8 \mathrm{mmHg}$ (Gráfico 1). Nos demais grupos, com qualquer dos três tipos de válvula anti-refluxo, ocorreu aumento pósoperatório das medidas relativas à pressão na ZAP. Nos grupos com válvula, a média de pressão na ZAP não apresentou diferença significativa quando comparada à préoperatória: as três modalidades foram eficazes no sentido de restaurar a ZAP. As comparações entre o valor médio aí obtido no grupo controle com o registrado em qualquer dos grupos com válvula demonstraram diferenças significativas, o que não foi observado quando comparados entre si os grupos com válvula (Gráfico 1).

A análise da variância e o teste de contraste de Scheffé demonstraram a eficácia das válvulas anti-refluxo no sentido de manter e até aumentar a pressão na ZAP, sendo significativa a diferença com o grupo controle, isto é, sem válvula. As três modalidades de válvulas foram igualmente eficientes, pois, não obstante o valor médio pós-operatório ter se mostrado maior com a válvula posterior $(44,8 \mathrm{mmHg})$, ele não diferiu de modo significante dos valores médios pós-operatórios obtidos com a válvula anterior $(40,9 \mathrm{mmHg})$, ou com a válvula lateral $(41,3 \mathrm{mmHg})$.

Dentre os métodos de verificação de esofagite, o exame histopatológico da peça mostrou ser o mais sensível, detectando maior número de lesões e em grau mais avançado. Observou-se que todos os animais do grupo controle tinham esofagite (Gráfico 2). Entretanto, em três casos, a endoscopia não demonstrou esofagite e em dois a biópsia endoscópica também não revelou esofagite.

Nos animais com válvula, quatro casos de esofagite foram reconhecidos através da endoscopia, seis casos pela biópsia e nove casos pelo exame histopatológico da peça (Gráfico 2). A esofagite encontrada nestes animais foi de menor extensão e de grau menos avançado do que nos do grupo controle. Porém, a comparação entre os grupos com válvula não encontrou diferenças quanto à esofagite seja através da endoscopia, seja pela histopatologia, da biópsia ou da peça.

\section{DISCUSSÃO}

Diversos estudos têm verificado abolição ou redução na ZAP após cardiomiotomia anterior ${ }^{22,23}$ ou lateral direita ${ }^{24,25}$. Neste estudo observou-se que a redução da ZAP no grupo controle foi de $15,8 \mathrm{mmHg}$, em média. Observouse que o afastamento das bordas da miotomia é semelhante após a cardiomiotomia ou cardiomiectomia devido à secção da musculatura circular que habitualmente é predominante e hipertrófica no megaesôfago.

Diversas publicações ressaltam a necessidade da utilização de válvulas após cardiomiotomias ou cardiomectomias e demonstram reaparecimento de zona de alta pressão após a confecção de válvulas parciais ou totais, por via abdominal ou torácica, pela técnica aberta ou laparoscópica. No presente estudo, observou-se que as três modalidades de válvulas (anterior, lateral e posterior) demonstraram eficácia na manutenção da pressão na ZAP. Em nosso meio, Gama-Rodrigues et al. ${ }^{26}$, em 1974, utilizando a técnica de LIND no tratamento do RGE, obtiveram discreto aumento na média da pressão basal no pós-operatório. Entretanto, Felix ${ }^{24}$, em 1987, empregando a técnica de Pinotti e de Chaib, não obteve efeito restaurador da pressão na ZAP no tratamento do megaesôfago chagásico.

Mosnier et al. ${ }^{28}$, em 1995, relataram excelentes resultados endoscópicos, pHmétricos e manométricos, empregando a válvula posterior de $270^{\circ}$ no tratamento laparoscópico do RGE.

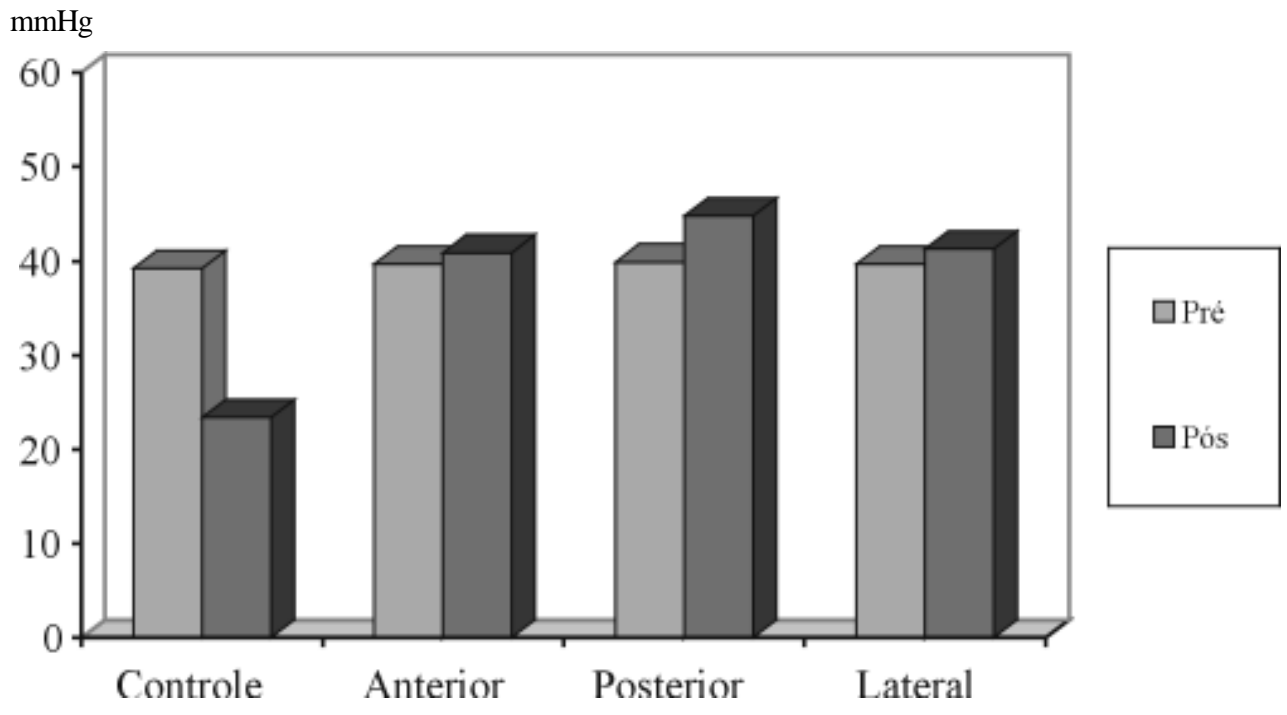

Gráfico 1 - Pressão média na zona de alta pressão segundo os grupos estudados $(n=25)$. 

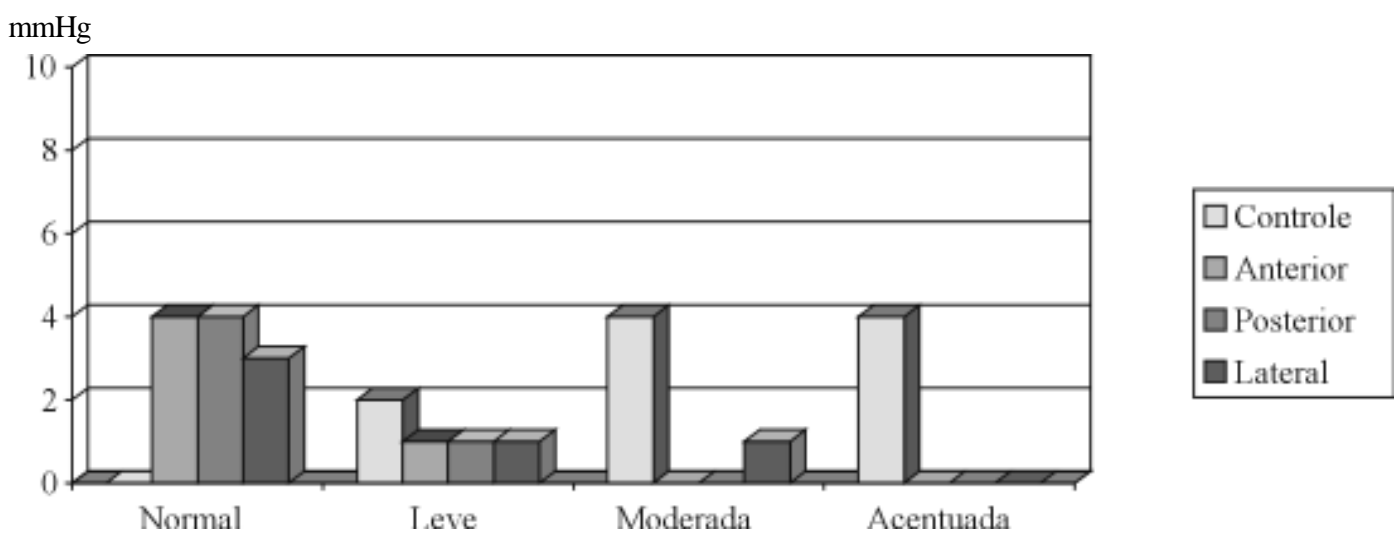

Gráfico 2 - Presença de esofagite ao estudo histopatológico da transição esofagogástrica ( $n=25)$.

Os critérios histopatológicos ${ }^{27}$ utilizados neste trabalho procuram detectar e ressaltar as alterações arquiteturais e citológicas do epitélio esofágico, conseqüente à agressão crônica causada pelo RGE.

As alterações encontradas com estes critérios histopatológicos nem sempre possuem tradução macroscópica detectável pela endoscopia, principalmente as de grau mínimo. Portanto, não existindo alterações macroscópicas, existe a possibilidade de as biópsias apresentarem ou não sinais histológicos de dano epitelial.

Procurou-se analisar em conjunto os diagnósticos endoscópico e histopatológico, com o intuito de observar de forma cuidadosa as alterações provocadas pelo RGE.

$\mathrm{O}$ exame histopatológico da peça dos animais com miotomia e sem válvula anti-refluxo demonstrou que todos apresentavam esofagite de graus variados. Entretanto, a endoscopia não detectou esofagite em dois animais.

Nos grupos com válvula, encontramos quatro animais com diagnóstico de esofagite ao exame endoscópico, e os grupos com válvula anterior e posterior apresentaram apenas um caso de esofagite leve cada um. Os animais do grupo com válvula lateral apresentaram dois casos de esofagite à endoscopia, sendo um de esofagite leve e um de esofagite moderada.

A análise de nossos resultados nos permite concluir que a cardiomiotomia ampla provoca RGE e conseqüente esofagite e inferir que, no cão, as válvulas anti-refluxo efetuadas (anterior, lateral e posterior) restauram um segmento de alta pressão após a cardiomiotomia, diminuem de maneira significante a incidência de refluxo, bem como amenizam as alterações secundárias à agressão crônica da mucosa esofágica.

Extrapolando, com ressalvas, para a cirurgia no ser humano, verificações experimentais sugerem que as fundoplicaturas anterior, posterior e lateral esquerda, após a cardiomiotomia anterior ampla, são equivalentes para a prevenção de RGE pós-operatório. Entretanto, a mais extensa dissecção necessária para a confecção da válvula posterior envolve maior risco de lesões acidentais. Assim é que Ferreira-Santos ${ }^{17}$, em 1982, a propósito da plicatura posterior, chama atenção para ligadura dos vasos curtos quando necessária, que pode resultar em lesão esplênica, e para a ampla abertura da parte alta do pequeno omento (necessária para a passagem retroesofágica da grande tuberosidade gástrica), que pode comprometer a integridade do ramo hepático do nervo vago anterior.

A vídeocirurgia por acesso abdominal ${ }^{22,25}$ ou torácico ${ }^{29}$ tem demonstrado resultados excelentes no tratamento do megaesôfago. Com o uso rotineiro da técnica laparoscópica no tratamento cirúrgico do megaesôfago, a possibilidade de utilização da gastrofundoplicatura através de duas fileiras de pontos, com envolvimento anterior (Lhotka-Dor-Goffi), posterior (LIND modificada) ou lateral (Lortat-Jacob-Ferreira-Santos) do esôfago, constituem opções terapêuticas que facilitam tecnicamente a confecção das válvulas, sendo as válvulas anteriores e anterolaterais preferidas pela maioria dos autores por cobrir a área da miotomia, evitando aderências pós-operatórias da mucosa que fica exposta quando praticada a válvula posterior.

Temos utilizado de rotina nestas situações, com bons resultados pós-operatórios, a técnica de Lhotka-Dor modificada por Goffi por laparoscopia a exemplo do que praticávamos quando da cirurgia aberta, considerando-a, após análise de 210 casos, ótima opção cirúrgica para o tratamento do megaesôfago chagásico não avançado, no que tange à abolição da disfagia e à prevenção do refluxo gastroesofágico pós-operatório.

A análise deste material nos permite concluir que:

1. a cardiomiotomia ampla reduziu de maneira significante a pressão na zona de alta pressão;

2. a cardiomiotomia ampla provocou refluxo gastroesofágico e conseqüente esofagite;

3. as válvulas anti-refluxo anterior, posterior e lateral diminuíram de maneira significante a incidência de refluxo e de esofagite;

4. houve restauração de um segmento de alta pressão após a confecção das válvulas anterior, posterior e lateral;

5. a comparação entre os três tipos de válvulas quanto à incidência de refluxo, restauração da ZAP e grau de esofagite não demonstrou diferença com significância estatística. 


\begin{abstract}
Objective: To compare three techniques of esophagogastrofundoplication - anterior, posterior and lateral-post cardiomyotomy, in dogs. Method: Pre-operative assessment included esophagoscopy plus biopsy of the mucosa, pH determination and manometry. The animals were divided into four groups: Group A: anterior anti-reflux valve $(n=5)$; group $P$ : posterior valve $(n=5)$; Group L: lateral valve $(n=5) ;$ Group C: control group, no fundoplication $(n=10)$. After five weeks, the animals were submitted to endoscopy plus biopsy, pHmetric and manometric examinations and histopathological analysis. Results: Histophatological examination detected esophagitis in every case of the control group and in nine cases of the groups with fundoplication. When present, esophagitis was milder in the valve groups than in the control group. The average value of the pressure in high pressure zone (HPZ) detected by manometric examination in the control group, was significantly reduced after cardiomyotomy $(39,2$ to $23.4 \mathrm{mmHg})$. In the groups with fundoplication, there was no significant difference between preoperative and postoperative mean values of the HPZ pressure. The values detected preoperatively in groups A, $P$ and L were 39.7, 39.9, and 39.7 $\mathrm{mmHg}$ respectively and 40.9, 44.8 and $41.3 \mathrm{mmHg}$ postoperatively. Conclusions: 1) cardiomyotomy causes gastroesophageal reflux and esophagitis; 2) any type of fundoplication valve, added to the myotomy, restores the lenght and the pressure of the HPZ=ZAP and reduces the incidence of reflux and esophagitis.
\end{abstract}

Key Words: Esophagogastrodundoplication; Cardiomiotomy; Anti-reflux valves.

\title{
REFERÊNCIAS
}

1. Heller E. Extramuskose cardioplastik beim Chronischen Cardiospasmus. Mit dilatation des oesophagus. Mitt Genzgeb.Med.Chir. 27:141-149, 1913.

2. Groeneveld FRB. Over cardiospasmus. Ned.Tijdschr. Geneeskd; 2: 1281-1286, 1918.

3. SengW. Cirurgia do esôfago. Bol.Soc.Cir.; 5; 128 - 132, 1922.

4. Mattos JO. Tratamento do megaesôfago (análise da cárdia) pela esfincterotomia cárdica. AMB, 4; 217-223, 1938.

5. Nemir Jr. P, Hawthorn MR. Physiologic basic of utilization of oesophagocardiomiotomy in treatment of achalasia. J. Thorac. Cardiovasc. Surg. 28: 247-258, 1954.

6. Ferreira-Santos RE. Tratamento cirúrgico da aperistalse esofágica (megaesôfago). Ribeirão Preto, Faculdade de Medicina, 1963. (Tese defendida no concurso para provimento efetivo da cadeira do Departamento de Cirurgia de Faculdade de Medicina de Ribeirão Preto da Universidade de São Paulo).

7. Ellis FH. Reflux after cardiomiotomy. Gut, 6:80, 1965.

8. Fyke Jr. e col. The gastroesophageal sphincter in healthy human beings. Gastroenterologia (Basel) 86; $135-$ $141,1956$.

9. Botha GSM, Astley R, Carre IJ. A combined cineradiographic and manometry study of the gastro-oesophageal junction. Lancet, 1; 659-662, 1957.

10. Pope CE. A dinamic test of sphincter strength: its aplication to the lower esophageal sphincter. Gastroenterology, 52: 779 - 786, 1967.

11. Bombeck CT e col. An experimental comparision of procedures for the operative correction of gastroesophageal reflux. Bull. Soc. Int. Chir. 30: 435-443, 1971.

12. Nissen E, Rosseti VM. Modern operation for hiatal hernia and reflux esophagitis: gastropexy and fundoplication. Arch. Surg. 120: 663-668, 1985.

13. Donahue PE e col. The floppy Nissen fundoplication: effective long-term control of pathologic reflux. Arch.Surg., 120: 663-668, 1985.
14. Lortat-Jacob JL e col. La prévention des hemorragies digestives aprés opération fe Heller. Ver. Ass. Franç.Chir., 58: 162-164, 1956

15. Goffi FS e col. Tratamento cirúrgico da esofagite por refluxo. In: Bastos ES. Tomos modernos da cirurgia. São Paulo. Procienx, 222-226, 1969.

16. Skinner DB, Belsey RHR. Surgical management of esophageal reflux and hiatus hernia: long-term results with 1030 patients. J. Thorac. Carciovasc. Surg. 53:33-54, 1967.

17. Ferreira-Santos RE. Hérnia hiatal e/ou esofagite de refluxo. Alguns aspectos importantes do diagnóstico e do tratamento. In: Nyhus LM, Waltell C. Cirurgia do estômago e do duodeno, $3^{\text {a }}$ - ed., tradução do Prof. Dr. Ruy Ferreira Santos. Rio de Janeiro. Interamericana, 1982. p. 547-569.

18. Jeckler J, Lhotka J. Modified Heller procedure to prevent post-operative reflux esophagitis in pacients with achalasia. Am. J. Surg., 113:251-254, 1967.

19. Dor J e col. L'interêt de la technique de Nissen modifiee dans la prevention du reflux aprés cardiomyotomie extra-muquese de Heller. Mém.Ac. Chir.; 1962, 88, p. 877.

20. Lind JF. Physiological repair of hiatus hernia - manometric study. Arch. Sur., 91:223, 1965.

21. Pinotti HW. Novas bases para o tratamento cirúrgico do megaesôfago: esofagocardiomiotomia com esofagofundogastropexia. Ver. Assoc. Med. Bras., 20: 331, 1974.

22. Morino M. Laparoscopic Heller cardiomyotomy with intraoperative manometry in the management of oesophageal achalasia. Int. Surg. 80(4), 332-5, 1995.

23. Peracchia A. Laparoscopic approach to esophageal achalasia. Am. J. Surg., 169(4): 424-427, 1995.

24. Felix VN. Contribuição ao conhecimento do mecanismo de ação da valvuloplastia esofagogástrica. São Paulo, Faculdade de Medicina da Universidade de São Paulo, 1987. 60p. (Tese de Doutorado). 
25. Felix VN. Tratamento cirúrgico do megaesôfago: Efeito da miotomia e da valvuloplastia sobre o esfíncter inferior do esôfago. Arq.Gastroenterol. 33 (1): 17-25, 1996.

26. Gama-Rodrigues JJ. Hérnia hiatal por deslizamento. Esofagofundogastropexia associada a hiatoplastia - avaliação clínica, morfológica e funcional. São Paulo, Faculdade de Medicina da Universidade de São Paulo, 1974. 57p. (Tese Livre-Docência).

27. Monsnier $\mathrm{H}$ e col. A $270^{\circ}$ degree laparoscopic posterior fundoplasty in treatment of gastroesophageal reflux. J. Am. Coll. Surg. 181(3):220-4, 1995.

28. Ismail Beigi $\mathrm{F}$ e col. Histological consequences of gastroesophageal reflux in man. Gastroenterology, 58:163174, 1970.
29. Pelegrini CA. Minimaly invasive surgery for gastroesophageal reflux disease. Am.J.Surg., 170: 614-618, 1994.

Endereço para correspondência:

Dr. Eduardo Crema

Rua Duque de Caxias, 165/301

38022-180 — Uberaba-MG 